\title{
Neutrino-deuteron reactions at solar neutrino energies
}

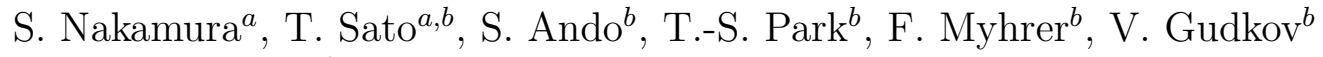 \\ and K. Kubodera ${ }^{b}$ \\ ${ }^{a}$ Department of Physics, Osaka University, Toyonaka, Osaka 560-0043, Japan \\ ${ }^{b}$ Department of Physics and Astronomy, University of South Carolina, Columbia, SC \\ 29208, USA
}

In interpreting the SNO experiments, accurate estimates of the $\nu d$ reaction cross sections are of great importance. In our recent work [ [], we have improved our previous calculation by updating some of its inputs and by incorporating the results of a recent effective-field-theoretical calculation. The new cross sections are slightly $(\sim 1 \%)$ larger than the previously reported values. It is reasonable to assign $1 \%$ uncertainty to the $\nu d$ cross sections reported here; this error estimate does not include radiative corrections.

\section{Introduction}

The establishment of the Sudbury Neutrino Observatory (SNO) has greatly increased the necessity of detailed theoretical studies of the neutrino-deuteron $(\nu d)$ reactions[ 1, 2, 3, 4, 5. At SNO, neutrino oscillations can be directly tested by measuring the solar electron-neutrino flux using the charged-current $(\mathrm{CC})$ reaction $\left(\nu_{e} d \rightarrow e^{-} p p\right)$ and the total solar neutrino flux using the neutral-current (NC) reaction, $\nu_{x} d \rightarrow \nu_{x} p n(x=e, \mu$ or $\tau)$. The recent SNO results[ 6] have given definitive evidence for neutrino oscillations. To make the best use of the existing and future SNO data, it is highly desirable to further improve the theoretical estimates of the $\nu d$ cross sections.

A traditional approach for describing nuclear electroweak processes consists in evaluating the contributions of 1-body impulse-approximation (IA) operators and 2-body exchange-current (EXC) operators defined in the Hilbert space of non-relativistic nuclear wave functions, with the EXC terms derived from one-boson exchange diagrams. We refer to this approach as SNPA (standard nuclear physics approach). The calculations of the $\nu d$ cross sections $\left(\sigma_{\nu d}\right)$ based on SNPA have been done by several authors [ 4$]$; the most recent work due to Nakamura et al. shall be referred to as NSGK [ [] and NETAL [ 1].

An alternative approach, effective field theory (EFT), has been applied to the $\nu d$ reaction by Butler et al.(BCK)[5]. The EFT lagrangian used by BCK involves one unknown low-energy constant (LEC), $L_{1 A}$, which BCK adjusted to optimize fit to the $\sigma_{\nu d}$ of NSGK. After this fine-tuning, the results of BCK were found to agree with those of NSGK within $1 \%$ over the entire solar-neutrino energy region. Very recently, Ando et al. have performed an EFT-motivated calculation of $\sigma_{\nu d}$ [ [3] using a method called EFT*; in this approach, originally proposed by Park et al.[ 7], the electroweak transition operators are derived with a cut-off scheme EFT, while the initial and final nuclear wave functions are obtained with the use of a realistic phenomenological $N N$ potential. The $\mathrm{EFT}^{*}$ lagrangian contains 
an unknown LEC, denoted by $\hat{d}^{R}$, which plays a role similar to $L_{1 A}$ in BCK. In EFT*, however, $\hat{d}^{R}$ can be determined directly from the tritium $\beta$-decay rate $\Gamma_{t}^{\beta}$, which allows a parameter-free calculation of the $\nu d$ cross sections.

We give here a concise account of the latest SNPA calculation of the $\nu d$ reaction carried out in NETAL [ 1]. The main points of improvement over NSGK are as follows. First, the updated value of the axial coupling constant $g_{A}$ is used. Secondly, the treatment of the axial-vector exchange current $\left(\boldsymbol{A}_{\mathrm{EXC}}\right)$ is improved. Since the $\nu d$ reaction in the solar-neutrino energy region is dominated by the Gamow-Teller (GT) transition, $\boldsymbol{A}_{\mathrm{EXC}}$ is a crucial ingredient that controls the accuracy of calculated cross sections. Among the various terms in $\boldsymbol{A}_{\mathrm{EXC}}$, the $\Delta$-excitation current is the most important one. NETAL uses the $\Delta$-excitation current determined in Ref. [9] to reproduce the experimental value

of $\Gamma_{t}^{\beta}$. Thirdly, NETAL employs the effective Fermi coupling constant $G_{F}^{\prime}$ that includes inner-radiative corrections instead of $G_{F}$ used in NSGK. Furthermore, the stability of theoretical estimates is investigated by comparing the new SNPA calculation with Ando et al.'s EFT* calculation [ 3].

\section{Formalism}

The interaction hamiltonian $\left(H_{W}\right)$ for a weak semileptonic process is given by the product of the hadron current $\left(J_{\lambda}\right)$ and the lepton current $\left(L^{\lambda}\right)$ as

$H_{W}^{X}=\frac{G_{F}^{X}}{\sqrt{2}} \int d \boldsymbol{x}\left[J_{\lambda}^{X}(\boldsymbol{x}) L^{\lambda}(\boldsymbol{x})+\right.$ h. c. $]$

with $X=\mathrm{CC}$ or NC, and $G_{F}^{C C}\left(G_{F}^{N C}\right)=G_{F}^{\prime} V_{u d}\left(G_{F}^{\prime}\right)$, where $G_{F}^{\prime}$ is the weak coupling constant determined from the $0^{+}-0^{+} \beta$-decay rates, and $V_{u d}$ is the K-M matrix element. The hadron current is the sum of the vector $\left(V_{\lambda}\right)$ and the axial current $\left(A_{\lambda}\right)$;

$J_{\lambda}^{C C}(\boldsymbol{x})=V_{\lambda}^{+}(\boldsymbol{x})+A_{\lambda}^{+}(\boldsymbol{x})$

for the $\mathrm{CC}$ reaction and

$J_{\lambda}^{N C}(\boldsymbol{x})=\left(1-2 \sin ^{2} \theta_{W}\right) V_{\lambda}^{3}(\boldsymbol{x})+A_{\lambda}^{3}(\boldsymbol{x})-2 \sin ^{2} \theta_{W} V_{\lambda}^{s}(\boldsymbol{x})$

for the NC reaction. Here $\theta_{W}$ is the Weinberg angle. The hadron current consists of the 1-body IA current and the 2-body EXC, whose explicit forms can be found in Ref. [1]. Other details of the calculation including multipole expansion and the cross section formula are given in Ref.[2]. For numerical results, NETAL used the deuteron and the $N N$ scattering wave functions generated with the ANLV18 potential [10].

\section{Results and Discussion}

The calculated total cross section for the $\mathrm{CC}$ reaction $\left(\sigma_{\nu d}^{C C}\right)$ is shown in Fig.1 as a function of the incident neutrino energy $\left(E_{\nu}\right)$. Although the cross section is dominated by the GT transition leading to the final ${ }^{1} S_{0} N N$ state, the transitions leading to higher partial waves are not totally negligible; they account for $1 \%$ and $4 \%$ of the reaction rate at $E_{\nu} \sim 10 \mathrm{MeV}$ and $E_{\nu} \sim 20 \mathrm{MeV}$, respectively. 


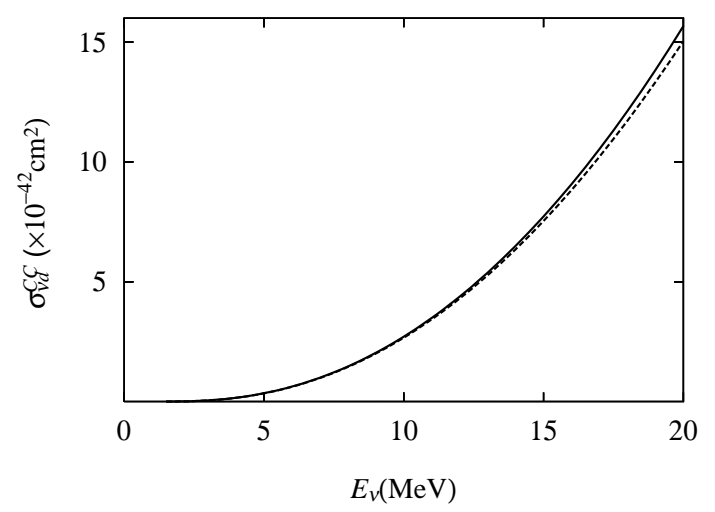

Figure 1. Total cross sections for the CC reaction. The solid- (dotted-) curve includes all $\left({ }^{1} S_{0}\right)$ partial waves of the final two-nucleon system.

\begin{tabular}{cccc}
\hline$E_{\nu}(\mathrm{MeV})$ & NETAL & NSGK & normalized \\
\hline 5 & 1.019 & 1.050 & 1.020 \\
10 & 1.023 & 1.059 & 1.024 \\
20 & 1.029 & 1.068 & 1.030 \\
\hline
\end{tabular}

Table 1

Contributions of EXC for the $\mathrm{CC}$ reaction. The ratio, $\sigma_{\nu d}^{C C}(\mathrm{IA}+\mathrm{EXC}) / \sigma_{\nu d}^{C C}(\mathrm{IA})$, is shown for NETAL (2nd column) and for NSGK (3rd column). The fourth column corresponds to the use of the "normalized" $\boldsymbol{A}_{\mathrm{EXC}}^{\mathrm{NSGK}}$ explained in the text.

\begin{tabular}{ccc}
\hline$E_{\nu}(\mathrm{MeV})$ & $\nu_{e} d \rightarrow e^{-} p p$ & $\nu d \rightarrow \nu p n$ \\
\hline 5 & 1.003 & 1.004 \\
10 & 1.001 & 1.003 \\
20 & 0.998 & 1.001 \\
\hline
\end{tabular}

Table 2

Comparison of SNPA and EFT*. The ratio $\sigma_{\nu d}\left(\mathrm{EFT}^{*}\right) / \sigma_{\nu d}(\mathrm{SNPA})$ is shown for the $\mathrm{CC}$ and $\mathrm{NC}$ reactions.

The ratio $\sigma_{\nu d}^{C C}(\mathrm{IA}+\mathrm{EXC}) / \sigma_{\nu d}^{C C}$ (IA) is shown in Table 1 for both NETAL and NSGK. In NETAL, the EXC contribution to $\sigma_{\nu d}^{C C}$ is $\sim 2 \%$, which is $\sim 3 \%$ smaller than the NSGK results. The strength of $\boldsymbol{A}_{\mathrm{EXC}}^{\mathrm{NSGK}}$ was determined by analyzing the $n p \rightarrow \gamma d$ reaction and assuming a quark-model relation between the axial vector and vector coupling constants for the $N \Delta$ transition. We note, however, that $\Gamma_{t}^{\beta}$ should give a much more direct constraint on $\boldsymbol{A}_{\mathrm{EXC}}$ than $\sigma(n p \rightarrow d \gamma)$, the latter being governed by the vector current. One way to gauge the sensitivity of $\sigma_{\nu d}$ to different choices of $\boldsymbol{A}_{\mathrm{EXC}}$ is to normalize the strength of $\boldsymbol{A}_{\mathrm{EXC}}^{\mathrm{NSGK}}$ near threshold to reproduce $\boldsymbol{A}_{\mathrm{EXC}}^{\mathrm{NETAL}}$. With this normalization applied, the difference between the two EXC models is reduced to $0.2 \%$; see the fourth column in Table 1. Thus it is the overall strength of $\boldsymbol{A}_{\mathrm{EXC}}$ that controls the low-energy $\nu d$ reactions; $\sigma_{\nu d}$ is insensitive to the detailed structure of $\boldsymbol{A}_{\mathrm{EXC}}$ once EXC is adjusted to reproduce $\Gamma_{t}^{\beta}$.

Comparison with an $\mathrm{EFT}^{*}$ calculation [ [3] provides a further test of the reliability of $\sigma_{\nu d}$ obtained in NETAL. It is sufficient to make this comparison for the reaction rate leading to the final ${ }^{1} S_{0}$ state. Table 2 shows the ratio $\sigma_{\nu d}\left(\mathrm{EFT}^{*}\right) / \sigma_{\nu d}(\mathrm{SNPA})$, from which we can conclude that SNPA and EFT* give identical results within $1 \%$ accuracy. This 
agreement proves the robustness of the theoretical estimates of $\sigma_{\nu d}$ obtained in NETAL.

With the improved inputs as described above, NETAL have obtained $\sigma_{\nu d}$ 's that are slightly larger $(\sim 1 \%)$ than those of NSGK. The $\sigma_{\nu d}$ 's in NETAL are considered to be reliable within $1 \%$ accuracy.

Besides the absolute value of $\sigma_{\nu d}$, the ratio $R=\sigma_{\nu d}^{N C} / \sigma_{\nu d}^{C C}$ is an important quantity for SNO experiments. Comparing the $R$ 's obtained in NETAL, NSGK and EFT*, we can assign $0.5 \%$ accuracy to the calculated $R$.

The radiative corrections are expected to affect $\sigma_{\nu d}$ at the level of a few per cent. NETAL only took into account a part of the radiative correction incorporated into $G_{F}^{\prime}$; the most recent estimation of the remaining radiative corrections can be found in Ref. [ 11.

A new experimental value of $g_{A}$ has been presented at this conference by Abele [ 12]. The reported value, $g_{A}=1.274$, is significantly larger than the current PDG value used by NETAL. We remark, however, that if the calculation of NETAL is repeated with the use of this new value of $g_{A}$, the resulting $\sigma_{\nu d}$ 's would be essentially unchanged. Since the strength of $\boldsymbol{A}_{\mathrm{EXC}}$ in NETAL is determined to reproduce $\Gamma_{t}^{\beta}$, an increase in $g_{A}$ is largely compensated by a decrease in $\boldsymbol{A}_{\mathrm{EXC}}$, leaving $\sigma_{\nu d}$ 's essentially unaffected.

This work is supported in part by the Japan Society for the Promotion of Science, Grant No. (c) 12640273, and by the US National Science Foundation, Grant No. PHY-9900756 and No. INT-9730847.

\section{REFERENCES}

1. S. Nakamura, T. Sato, S. Ando, T.-S. Park, F. Myhrer, V. Gudkov and K. Kubodera, Nucl. Phys. A707 (2002) 561.

2. S. Nakamura, T. Sato, V. Gudkov and K. Kubodera, Phys. Rev. C 63 (2001) 034617.

3. S. Ando, Y. H. Song, T.-S. Park, H. W. Fearing, and K. Kubodera, nucl-th/0206001.

4. N. Tatara, Y. Kohyama and K. Kubodera, Phys. Rev. C 42 (1990) 1694; S. Ying, W. C. Haxton and E. M. Henley, Phys. Rev. C 45 (1992) 1982.

5. M. Butler and J.-W. Chen, Nucl. Phys. A675 (2000) 575; M. Butler, J.-W. Chen and X. Kong, Phys. Rev. C 63 (2001) 035501.

6. Q. Ahmad et al., Phys. Rev. Lett. 87 (2001) 071301; 89 (2002) 011301; 89 (2002) 011302.

7. T.-S. Park, K. Kubodera, D.-P. Min and M. Rho, Nucl. Phys. A684 (2001) 101, and references therein.

8. J. F. Beacom and S. J. Parke, Phys. Rev. D 64 (2001) 091302.

9. R. Schiavilla, V. G. J. Stoks, W. Glöckle, H. Kamada, A. Nogga, J. Carlson, R. Machleidt, V. R. Pandharipande, R. B. Wiringa, A. Kievsky, S. Rosati and M. Viviani, Phys. Rev. C 58 (1998) 1263.

10. R. B. Wiringa, V. G. J. Stoks and R. Schiavilla, Phys. Rev. C 51 (1995) 38.

11. A. Kurylov, M. J. Ramsey-Musolf and P. Vogel, Phys. Rev. C 65 (2002) 05501.

12. H. Abele et al., Phys. Rev. Lett. 88 (2002) 211801; H. Abele, talk at this Conference. 